\title{
The Correlation between U.S. Tornadoes and Pacific Sea Surface Temperatures
}

\author{
CAREN MARZBAN \\ National Severe Storms Laboratory, Cooperative Institute for Mesoscale and Meteorological Studies, and \\ Department of Physics, University of Oklahoma, Norman, Oklahoma \\ JOSEPH T. SCHAEFER \\ Storm Prediction Center, Norman, Oklahoma
}

(Manuscript received 10 December 1999, in final form 16 August 2000)

\begin{abstract}
The correlation between tornadic activity in several regions of the United States and the monthly mean sea surface temperature over four zones in the tropical Pacific Ocean is examined. Tornadic activity is gauged with two mostly independent measures: the number of tornadoes per month, and the number of tornadic days per month. Within the assumptions set forth for the analysis, it is found that there appears to exist a statistically significant but very weak correlation between sea surface temperature in the Pacific Ocean and tornadic activity in the United States, with the strength and significance of the correlation depending on the coordinates at which the sea surface temperatures are assessed and the geographic region of the United States. The strongest evidence found is for the correlation between the number of days with strong and violent (F2 and greater) tornadoes in an area that runs from Illinois to the Atlantic Coast, and Kentucky to Canada and a cool sea surface temperature in the central tropical Pacific. However, there is only about a $53 \%$ chance of this relationship occurring in a specific month.
\end{abstract}

\section{Introduction}

Originally the term El Niño was used to describe the southward weak warm coastal current that develops along the coast of Ecuador each year during the Christmas season (Rasmusson and Carpenter 1982). However, over the last three decades, the meaning of El Niño has been subtly altered to denote the occasional large-scale presence of warm surface water across much of the eastern and central Pacific (e.g., Ramage 1975). Contrasting periods showing the presence of large-scale cool surface waters in the Tropical Pacific have come to be called La Niña.

The sea surface temperature pattern directly impacts the atmospheric pressure distribution over the tropical Pacific. During El Niño, or warm periods, surface pressure is lower than normal over the eastern tropical Pacific and higher than normal over northern Australia and Indonesia. These pressure patterns reverse themselves during the cool La Niña. This pressure variation is known as the Southern Oscillation (Rasmusson and Carpenter 1982). Rainfall patterns over the tropical Pacific exhibit a variation that is opposite in sign from that of

Corresponding author address: Caren Marzban, NRCSE, Box 354323, University of Washington, Seattle, WA 98195.

E-mail: marzban@nssl.noaa.gov pressure oscillation. During El Niño (La Niña), abnormally dry (wet) conditions occur over northern Australia, Indonesia, and the Philippines, with wetter (dryer) conditions prevailing over the west coasts of tropical North and South America.

The changes in the Tropics lead to shifts in the position of atmospheric circulation features. The jet stream over the middle and eastern Pacific Ocean is stronger than normal during an El Niño and weaker during a La Niña. Because of these teleconnections, El Niño/La Niña impacts the midlatitude weather in distant areas of the globe. Over the contiguous United States, El Niño episodes are associated with cool and wet winters in the gulf coast states, wet winters in southern California, and wet summers in the northern Rockies. In contrast, La Niña is associated with warm, dry winters from the southwestern states across the gulf coast states, and cool wet winters in the northwest (Halpert and Ropelewski 1987, 1992; Ropelewski and Halpert 1989).

Possible relationships between El Niño/La Niña and the occurrence of tornadoes in the contiguous United States has been the topic of several informal papers. The methods employed and the conclusions have been quite varied. Bove (1998) compared the annual number of reported tornadoes in $1.25^{\circ}$ latitude-longitude squares across the eastern two-thirds of the United States to the existence during the previous autumn of an El Niño/La 
Niña as determined by the Japan Meteorological Agency index. Resampling techniques were used to expand the tornado database. His analysis indicated that during the period February through July, El Niño years had a large decrease in the number of tornadoes in "Tornado Alley" (central Missouri through western Kansas, and south Texas through South Dakota), Arkansas, Louisiana, and Iowa. However, the Ohio and Tennessee River valleys experience a large increase during La Niña. He also found that over the Florida peninsula tornado activity is decreased during both El Niño and La Niña years as compared to neutral years. Browning (1998) also noted that on the average northwest Missouri has more tornadoes in La Niña years than in El Niño years; he simply counted the number of storms reported each year in northwest Missouri and compared them to the list of El Niño/La Niña years kept by the National Oceanic and Atmospheric Administration's Climate Diagnostic Center. In contrast to the tornado results, he found that both hailstorms and windstorms are more prevalent in El Niño years than La Niña years.

Somewhat different results were found by Agee and Zurn-Birkhimer (1998) through an examination of the ratio of the number of tornadoes in strong El Niño years to the number of tornadoes in La Niña years on a stateby-state basis. Their analysis indicated that Texas, Oklahoma, Missouri, Colorado, and New Mexico receive more tornadoes during strong El Niño years. However, as in the first study noted above, they found that the area from Iowa through the Carolinas, and Tennessee through Ohio has the most tornadoes during La Niña Years. Hagemeyer (1998) studied Florida tornadoes and noted that strong El Niños of the magnitude of 1983 and 1998 increase the chance of strong and violent weather in Florida.

Schaefer and Tatom (1998) determined the presence of El Niño/La Niña from the mean sea surface temperature (SST) in the strip $5^{\circ} \mathrm{N}$ to $5^{\circ} \mathrm{S}$ and $180^{\circ}$ to $150^{\circ} \mathrm{W}$. A Kruskal-Wallis $H$ test was then used to see if any difference in tornado counts exists between El Niño, La Niña, and neutral years. The annual number of tornadoes and the annual number of strong and violent tornadoes were both tested. Also the entire contiguous United States, and three subareas (Tornado Alley, the Mideastern United States, and Florida) were considered. All six of these combinations failed to have significance at the $99 \%$ level. They concluded that, with the data available, one could not state with confidence that El Niño/ La Niña had any effect on tornado or strong tornado activity.

These differing results are a reflection of the imprecise definition of the terms El Niño and La Niña. There are even conflicts in exactly which years are El Niño affected and which ones are La Niña affected (e.g., Bove 1998; Schaefer and Tatom 1998). This is compounded by ambiguities due to 1) different behavior of the sea surface temperature in different regions of the Pacific Ocean, 2) different methods of assessing and identifying an unusually warm or cold season, and 3) the possibility that the duration of a cold or warm phase does not coincide with the length of a year; a warm phase may begin in June of one year and last until some month in the following year.

In this paper, to attend to the first ambiguity, the mean monthly SST over four different zones in the Pacific is considered. The SSTs for the four zones are labeled as SST1, SST2, SST3, and SST4 (Fig. 1), corresponding to the following (Climate Prediction Center 1999):

- SST1: $0^{\circ}-10^{\circ} \mathrm{S}, 90^{\circ}-80^{\circ} \mathrm{W}$

- SST2: $5^{\circ} \mathrm{N}-5^{\circ} \mathrm{S}, 150^{\circ}-90^{\circ} \mathrm{W}$;

- SST3: $5^{\circ} \mathrm{N}-5^{\circ} \mathrm{S}, 170^{\circ}-120^{\circ} \mathrm{W}$; and

- SST4: $5^{\circ} \mathrm{N}-5^{\circ} \mathrm{S}, 160^{\circ} \mathrm{E}-150^{\circ} \mathrm{W}$.

The SST1 corresponds to the coastal Pacific, northwest of South America, generally the area where the term El Niño was first coined. SST2, SST3, and SST4 are overlapping zones that straddle the equator and extend progressively westward in the Pacific. SST2 is roughly the eastern equatorial Pacific, while SST4 is approximately the central equatorial Pacific. The four SSTs are often referred to as Niño-1+2, Niño-3, Niño-3.4, and Niño-4, respectively.

The second ambiguity is one that cannot be eliminated. To define what is meant by unusually warm or cold, one must first define what is meant by "usual." The latter, however, is often unknown and can only be inferred from a model. ${ }^{1}$ Then, the ambiguity in the definition of a warm or cold season is a direct consequence of the absence of a unique model describing the phenomenon. In other words, different models of the usual lead to different notions of the anomalous. As such, any conclusions based on SST anomalies are apt to be contingent on the assumptions underlying some model. In this paper, it is assumed that the usual component of the SSTs (and the tornadic activity) is one that can be filtered out by what is called seasonal differencing (see below). Therefore, the anomalies examined in this paper are specific to this type of filter/model.

Finally, to attend to the third ambiguity and to distance the analysis from the notion of an El Niño or La Niña year, the time unit of analysis will be the calendar month. The data span the 588 months from January 1950 to December 1998.

To measure tornadic activity, two metrics are commonly employed: the number of tornadoes per month and the number of tornadic days per month. In this article, although a slight emphasis is placed on the former when the data are examined directly, both measures

\footnotetext{
${ }^{1}$ Here, a model refers to a mathematical/statistical model underlying data, not a theoretical model involving the physical processes. Examples of the former include a regression fit to linear data, longterm averages and the deviations (anomalies) therefrom, deviations from a sinusoidal fit to a periodic signal, or other filtered signals (with the filter constituting a model).
} 


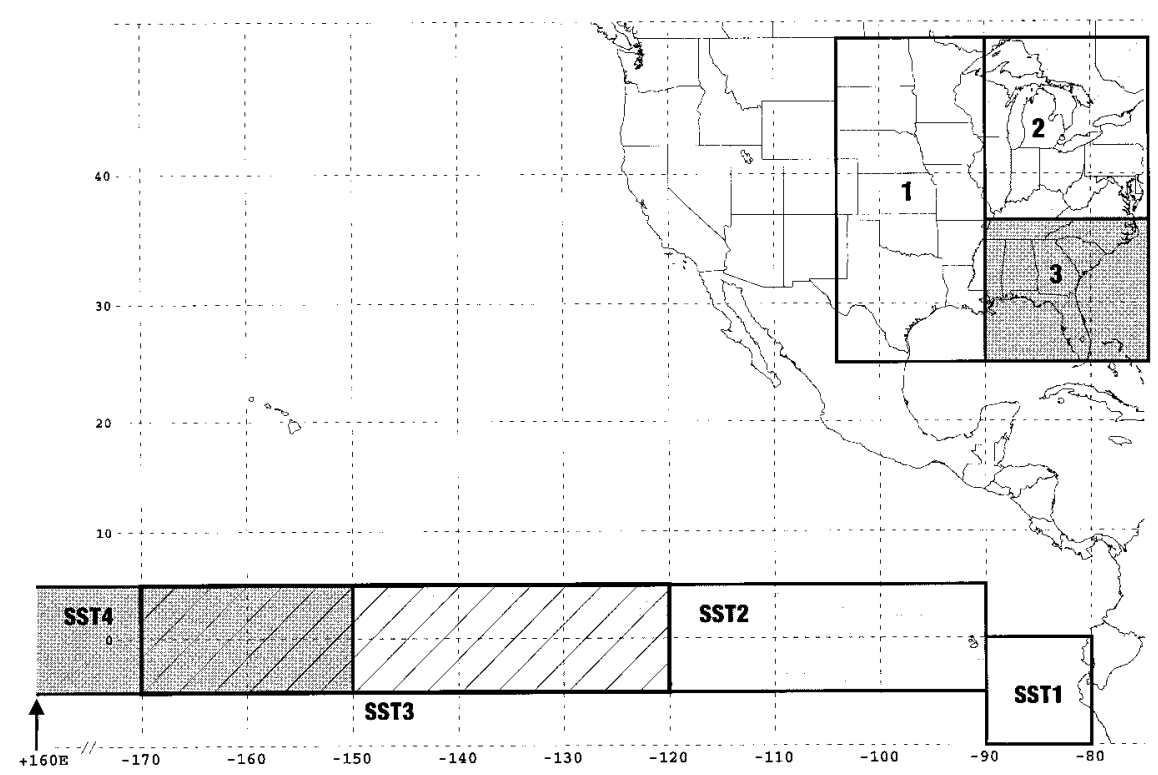

FIG. 1. The three regions of the United States, and the four zones in the Pacific.

are considered when the correlation between SST and tornadic activity is examined.

Given the effect of El Niño and La Niña on the position of the jet stream, it is conceivable that SST can have a different effect on tornadic activity depending on the particular geographic region wherein the tornadoes occur. Consequently, in addition to examining the correlation between SST and the nationwide tornadic activity, three distinct regions of the United States are also considered; they are defined as (Fig. 1)

- Region 1: The United States between $90^{\circ}$ and $105^{\circ} \mathrm{W}$,

- Region 2: The United States east of $90^{\circ} \mathrm{W}$ and north of $36.5^{\circ} \mathrm{N}$, and

- Region 3: The United States east of $90^{\circ} \mathrm{W}$ and south of $36.5^{\circ} \mathrm{N}$.

Region 1 consists of the Mississippi and Missouri Valley and corresponds to the area classically thought of as Tornado Alley. Region 2 runs from Kentucky and Virginia northward and includes the Ohio Valley (i.e., the northeast). Region 3 is the southeast, running from Tennessee and North Carolina southward. These regions were selected to be large enough so that monthly tornado counts would typically be nonzero, but small enough to show the subregional variability that is an essential feature of tornado climatology.

Also, because of the SST-jet stream relationship, it is possible that the correlation between SST and tornadic activity depends on tornadic strength. For this reason, in addition to tornadoes of all strength, the correlation between SST and tornadoes of strength F2 or higher is also analyzed separately. Henceforth, we shall refer to the latter as "strong and violent" tornadoes.

The analysis is done in the context of statistical hy- pothesis testing. In other words, every correlation between SST and tornadic activity computed from the sample will be subjected to the test that it is in fact zero (the null hypothesis). A statistical test is then performed to assess the confidence in any evidence to the contrary provided by the data. Each hypothesis is a statement regarding the correlation, but the choice of the measure of correlation is not unique. In this article, a nonparametric measure called Kendall's $\tau$ is considered (Press et al. 1986; Wilcox 1996). The appropriate test for this measure is the standard $z$ test. Since $\tau$ is a nonparametric statistic it assumes nothing about the distribution of the errors.

The outline of the paper is as follows: Section 2 provides a cursory view of the data in the form of time trends, distribution plots, and scatterplots. The measure of correlation and some basics of statistical hypothesis testing are provided in section 3 . In section 4 the method of analysis is outlined; the subsections address the preprocessing steps necessary for the final analysis. The results appear in section 5, followed by a summary and discussion in section 6 .

\section{Data}

The two variables under scrutiny are SST and tornadic activity, with the latter gauged in terms of two measures: the number of tornadoes per month and the number of tornadic days per month. The number of tornadoes as a function of intensity and for the three regions of the United States is available from the Storm Prediction 

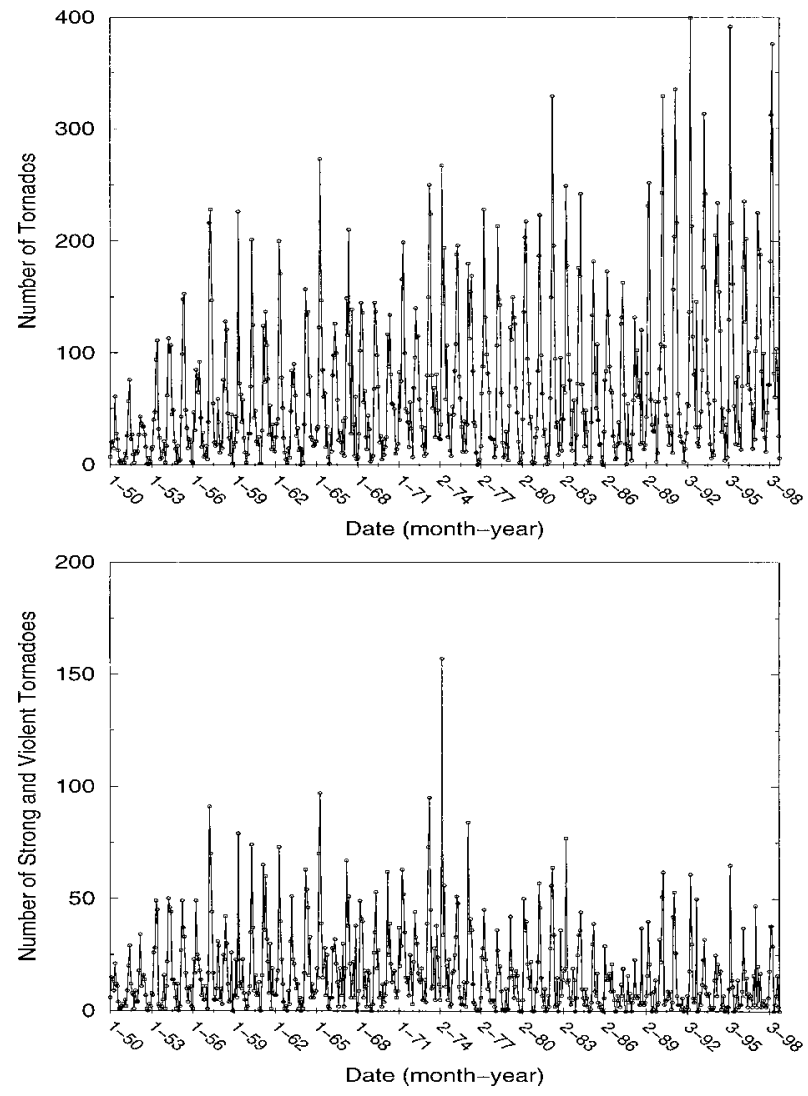

FIG. 2. The number of tornadoes as a function of time (i.e., month), for all tornadoes (F0-F5; top), and strong and violent tornadoes (F2F5; bottom), from Jan 1950 to Dec 1998.

Center for every month between the years 1950 and $1998 .^{2}$

A monthly mean sea surface temperature for the four Pacific zones calculated by a $1^{\circ}$ grid optimum interpolation analysis (Reynolds and Smith 1994) can be obtained from the CPC. The availability of these data allows for a direct examination of the correlation between tornadic activity and SST.

The number of tornadoes per month as a function of time (i.e., month) is displayed in Fig. 2; the top panel is for tornadoes of all strength, while the bottom panel pertains to strong and violent tornadoes (i.e., of strength F2 or higher).

The distribution of the frequencies is shown in Fig. 3. It is seen that the distributions peak to the left and are therefore neither normal nor bell-shaped. This has some indirect consequences in the statistical tests of the hypotheses. Performing a least squares linear fit calls for no assumptions regarding the distribution of the variables; however, to test the hypothesis that the slope of

\footnotetext{
${ }^{2}$ Some of the data can be found at http://www.spc.noaa.gov/ archive/tornadoes/. Updates occur on a time available basis. The total $\mathrm{SPC}$ dataset is available upon request.
}
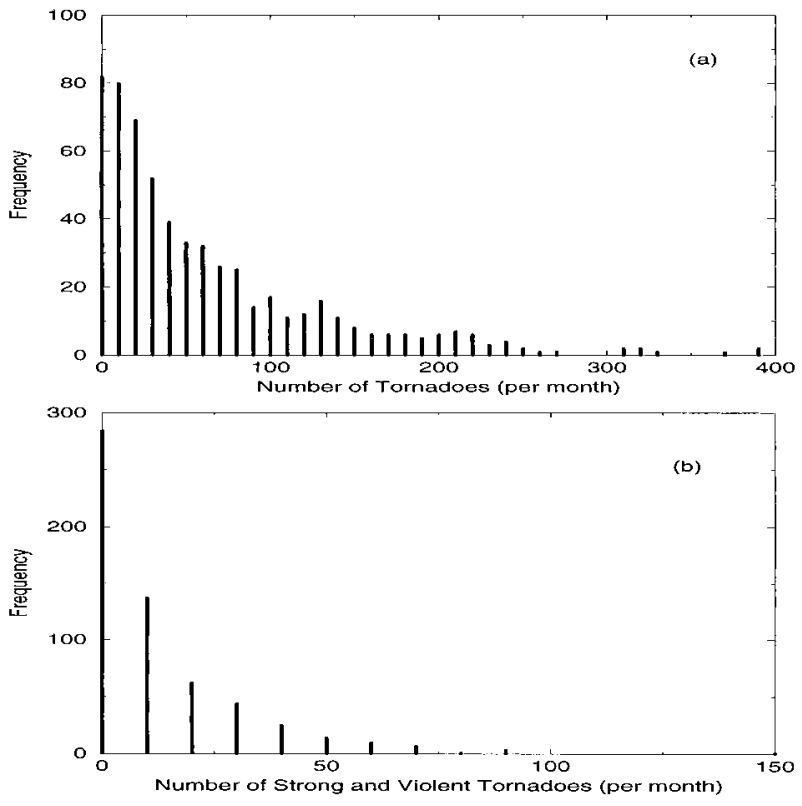

FIG. 3. The distribution of (a) all tornadoes and (b) strong and violent tornadoes. (bin size $=10$ ).

the fit is zero requires making assumptions regarding the distribution of errors (i.e., the difference between the dependent variable and the predicted value). For instance, a $t$ test of the hypothesis that the slope is zero requires the errors to be normally distributed. In the present case, however, since the dependent variable (i.e., tornadic activity) is not normally distributed, it is likely that the errors too will not be normally distributed. This can in fact be confirmed by examining the distribution of the residuals. As such, the $t$ test may fail. For this reason a nonparametric test, such as Kendall's $\tau$ (one of the so-called rank-based tests) is more suitable.

The distribution of the number of strong and violent tornadoes (Fig. 3b) resembles that for all tornadoes (Fig. $3 a)$. It is worth noting the average and the standard deviation of the number of strong and violent tornadoes for the three geographic regions; the averages are 9.6, 3.4 , and 3.1, and the standard deviations are 12.7, 5.7, and 4.8 , respectively. Clearly, region 1 is not only the most active in terms of strong and violent tornadic frequency, it is also the region with the most varied range of strong and violent tornadic frequency. Regions 2 and 3 are comparable both in terms of the average and the standard deviation of strong and violent tornadic frequency.

The distribution of the four SSTs is shown in Fig. 4. They are mostly bell-shaped, although they range over somewhat different temperatures. SST1 varies over the widest range (18.8-29.14), while SST4 has the narrowest range of temperature variations (26.53-29.78).

\section{Measure of correlation}

As mentioned previously, the measure of correlation considered is Kendall's $\tau$ (Press et al. 1986; Wilcox 

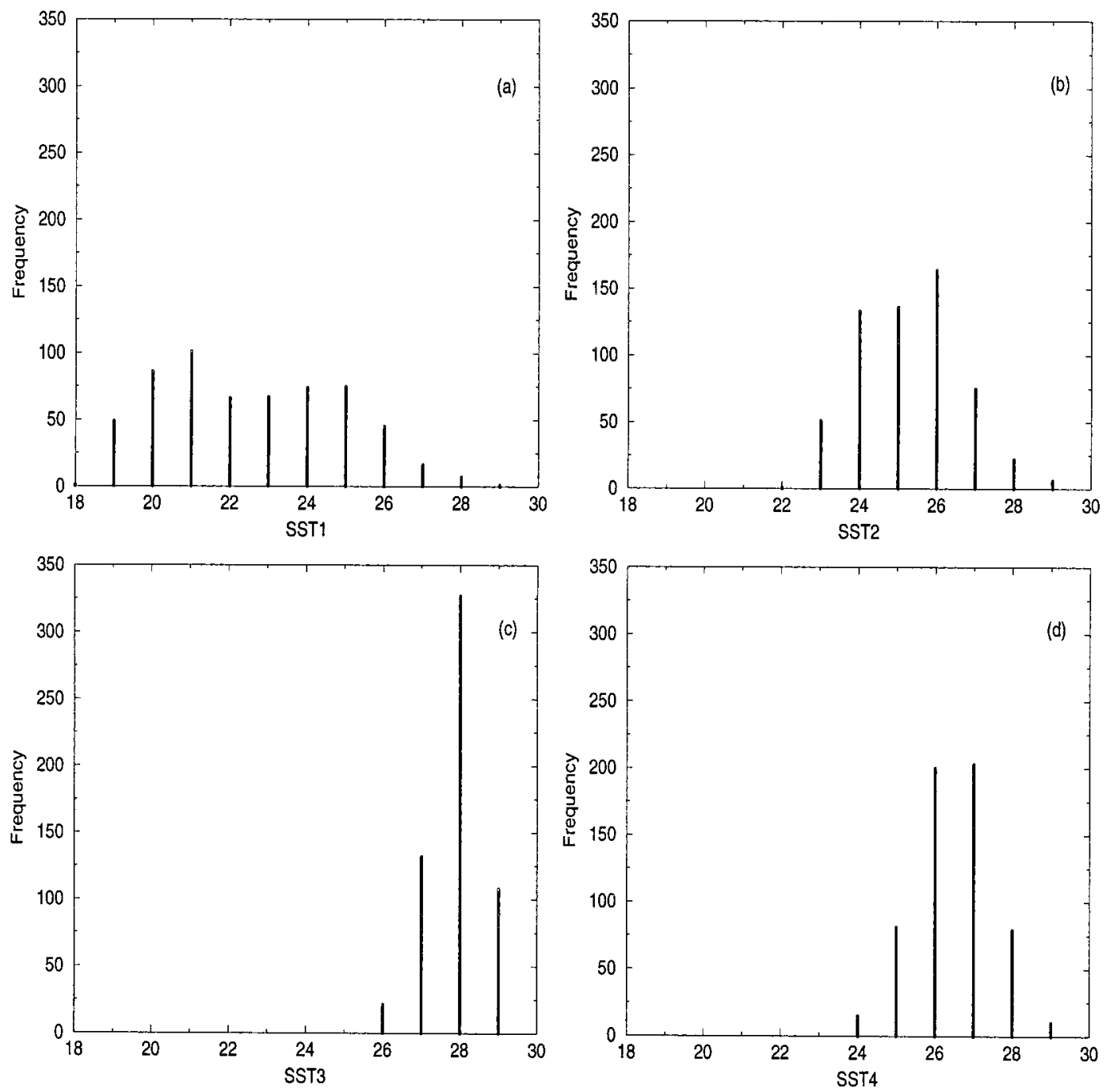

FIG. 4. The distribution of the four SSTs. The corresponding regions are (a) the coastal Pacific northwest of South America (SST1), (b) eastern equatorial Pacific (SST2), (c) east-central equatorial Pacific (SST3), and (d) central equatorial Pacific (SST4) $\left(\right.$ bin size $=1.0^{\circ}$ ).

1996). It is a measure of the monotonic relation (i.e., increasing or decreasing relations of any nature, linear or nonlinear) between a pair of variables. Its definition is based on the notion of concordant and discordant pairs. Concordant (discordant) pairs of data $(x, y)$ are those for which a larger $x$ is associated with a larger (smaller) $y$. Kendall's $\tau$ is defined as the difference between the proportion of pairs that are concordant and the proportion of pairs that are discordant.

With $n$ being the sample size and $p$ denoting the total number of pairs, then $p=n(n-1) / 2$, and $\tau$ is given by

$$
\begin{aligned}
\tau & =\frac{\text { concordant }- \text { discordant }}{p} \\
& =\frac{2 \text { (concordant }- \text { discordant })}{n(n-1)} .
\end{aligned}
$$

The value of $\tau$ ranges between +1 and $-1(|\tau| \leq 1)$. When $\tau>0$ more pairs are concordant (positive correlation), and when $\tau<0$ more pairs are discordant (negative correlation). If $\tau=0$, the number of concordant and discordant pairs is equal, and there is no correlation. Since the percentage of concordant and discordant pairs must sum to 1 , one can compute the percentage of concordant pairs as $100(1+\tau) / 2$. For example, if $\tau=0.5$, then $75 \%$ of the pairs are concordant. For a weaker correlation like $\tau=0.1,55 \%$ of the pairs are concordant (and $45 \%$ are discordant).

For any quantity computed from a sample it is important to compute a statistic that gauges whether the computed value can be generalized to the population and with what level of confidence. That issue falls in the realm of hypothesis testing. In the present context, the null hypothesis is that $\tau=0$, and a statistical test 
will be performed to test that hypothesis. It can be shown (Wilcox 1996) that the appropriate test for Kendall's $\tau$ is based on the $z$ statistic, with

$$
z=\frac{\tau}{\sqrt{\sigma^{2}}}
$$

where

$$
\sigma^{2}=\frac{2(2 n+5)}{9 n(n-1)} .
$$

The statistic $z$ follows the standard normal distribution as tabulated in most statistics texts and computer programs. The appendix offers a simple illustration of these formulas.

If $|z|>z_{1-\alpha / 2}$, then one can reject the null hypothesis; otherwise, there is insufficient evidence provided by the data for rejecting the possibility that the population $\tau$ is, in fact, zero. According to the tabulated values any $|z|$ larger than 2.575 provides sufficient evidence for claiming that the population $\tau$ is nonzero at the $\alpha=$ 0.01 level. This value of $\alpha$ corresponds to a $99 \%$ probability that the sample value of $\tau$ lies within a narrow range of the population value. ${ }^{3}$ It also means that only $1 \%$ of the time the null hypothesis will be incorrectly rejected (type I error). A few other conventional probabilities are $95 \%$ probability, corresponding to a $z$ value of $1.960(\alpha=0.05)$, and $90 \%$ probability corresponding to $z=1.645(\alpha=0.1)$. Usually any value of $|z|$ less than 1.645 (i.e., less than $90 \%$ confidence) is considered to provide insufficient evidence for rejecting the null hypothesis. Although the binary process of rejecting or not rejecting a hypothesis is inherent in hypothesis testing, it is preferable not to declare a hypothesis as true or false, and instead to simply report the confidence in its truth in the form of the $z$ value itself. For this reason, when possible and justified, we will refrain from making binary assertions (significant or not). However, when a binary decision is important, we shall make it.

\section{Method}

Testing for the existence of a correlation between two variables calls for some preprocessing. In particular, if the two variables have some nontrivial dependence on time, for example, if one is dealing with two time series (as is the case in this article), then it is necessary to

\footnotetext{
${ }^{3}$ Confidence intervals are more insightful; however, given that we are dealing with four geographic regions, four SSTs, two types of tornadoes (of any strength, and strong and violent), and two measures of tornadic activity, there are 64 correlations that must be examined. Producing confidence intervals for each of those 64 numbers can obfuscate the presentation. For that reason only the values of the $z$ statistic itself will be reported in this article.
}

remove trends and all traces of periodicity. ${ }^{4}$ Trends may dominate the time series to such a great extent as to obfuscate the underlying function of interest. Periodicity may lead to unreasonably large correlations between two variables. This occurs because the correlation may be a simple consequence of the periodic nature of the two variables rather than of a true correlation between the two. The following sections examine linear trends and the cycles in the data, and a method for eliminating both.

\section{a. Trends}

As seen from Fig. 2 (top panel), overall tornadic frequency shows an increasing trend with time. In fact, Kendall's $\tau$ can be employed to assess this trend in the unprocessed data. The value of $\tau$ and its associated $z$ is 0.17 and 6.24 , respectively. This suggests a moderate (about $58 \%$ of the pairs being concordant) and highly significant trend. The increase in tornado reports is most likely a manifestation of changes in society (e.g., increasing population, urbanization of previously sparsely populated areas such as central Florida, increased mobility, easier communication/cellular phones) and in improvements in detecting tornadoes (e.g., the start of a warning verification program in the early 1980s, the implementation of a national Doppler radar network in the early 1990s), rather than a change in the tornado occurrence rate (Grazulis et al. 1993).

By contrast, the number of strong and violent tornadoes shows a decreasing trend. In fact, one finds $\tau$ $=-0.12$ and $z=-3.70$, again suggesting a moderate and highly significant trend. This negative trend in the annual number of F2 and greater tornadoes reported is most likely related to the way the intensity of tornadoes was rated (Schaefer and Edwards 1999). Virtually all $F$-scale ratings from 1950 through the mid-1970s were based upon newspaper reviews. In contrast, since the late 1970s, National Weather Service personnel visit the site of all strong and violent tornadoes immediately after the storm and do a personal damage survey. These personnel visits have decreased the impact of the news media's fascination with extreme devastation. It is likely that the number of strong and violent tornadoes were overestimated during the earlier years of the database.

The four SSTs also have linear trends in time. Their $(\tau, z)$ values are $(0.04,1.41),(0.06,2.27),(0.08,3.07)$, and $(0.19,6.84)$, respectively. In other words, SST1 exhibits the smallest trend with the lowest confidence, while SST4 has the strongest and the most significant trend. SST3 and SST2 have weak trends at moderate significance levels.

However, any trend in either the number of tornadoes

\footnotetext{
${ }^{4}$ In the present case, it is sufficient to eliminate only the seasonal cycle. It is also assumed that the trend is linear.
} 
TABLE 1. Kendall's $\tau$ and its $z$ statistic, $(\tau, z)$, for the correlation between the number of strong and violent tornadoes and SST, prior to the removal of the seasonal factor.

\begin{tabular}{|c|c|c|c|c|}
\hline & SST1 & SST2 & SST3 & SST4 \\
\hline Contiguous United States & $(0.10,3.51)$ & $(0.21,7.56)$ & $(0.17,6.21)$ & $(-0.004,-0.13)$ \\
\hline Region 1: Tornado Alley & $(0.07,2.49)$ & $(0.19,6.98)$ & $(0.17,6.34)$ & $(0.02,0.77)$ \\
\hline Region 2: Northeast United States & $(0.10,1.00)$ & $(0.69,3.84)$ & $(0.80,3.35)$ & $(0.09,0.25)$ \\
\hline Region 3: Southeast United States & $(0.21,7.69)$ & $(0.19,6.72)$ & $(0.08,2.74)$ & $(-0.07,-2.39)$ \\
\hline
\end{tabular}

or SSTs will violate one of the basic assumptions of most methods of time series analysis, namely, stationarity (Masters 1995). Therefore, it is necessary to remove (i.e., filter out) the trends from the data. Fortuitously, the method employed for removing the seasonal cycles (below) also removes all linear trends. As such, it is not necessary to explicitly detrend the data prior to the removal of the seasonal factor.

\section{b. Seasons}

A variety of correlations exist in the dataset. Some of the correlations must be identified prior to the application of a test, while the identification of other correlations can aid in a better understanding of the underlying processes. For instance, the seasonal periodicity of SST is a type of (auto-) correlation that violates a basic assumption of statistical tests, namely that of the independence of the observations. Tornadic activity also has a seasonal cycle, and so any examination of the correlation between tornadoes and SST is apt to be dominated by the periodic nature of each variable. Therefore, the seasonal dependence must by filtered out of each variable.

One method for filtering time series data is differencing (Jenkins and Watts 1969, p. 296; Hamming 1983, p. 46; Masters 1995, p. 251), a specific instance of which is seasonal differencing (Masters 1995, p. 261). The method is quite simple and straightforward; one simply subtracts from each observation the value exactly one cycle earlier. For instance, the number of tornadoes in January 1950 is subtracted from the number of tornadoes in January 1951, and the number of tornadoes in February 1950 is subtracted from that of February 1951, etc., for all the months in the data. It can be shown that this routine eliminates the fundamental, all harmonics, and any linear trend (Masters 1995).

\section{Results}

To illustrate how results based on data containing a seasonal dependence can be extremely misleading, Table 1 shows the values of Kendall's $\tau$ and the corresponding $z$ statistic for the correlation between the number of strong and violent tornadoes in various geographic regions with the four SSTs. According to the $z$ values in the table, almost all the results are highly statistically significant; recall that a $|z|$ value larger than 2.575 is considered statistically significant at the 99\% level $(\alpha$
$=0.01)$. The extreme values are $(\tau, z)=(0.21,7.69)$ and $(\tau, z)=(-0.07,-2.39)$, which correspond to the most significant positive and negative correlations, respectively. This suggests that the correlation between SST1 and the number of strong and violent tornadoes in region 3 (the southeast) is positive, relatively strong (with about $60 \%$ of the pairs being concordant), and significant, while SST4 has a weaker and negative correlation with strong and violent tornadoes occurring in the same region. This would correspond to an El Niño and a La Niña effect, respectively. However, as mentioned previously, the periodic nature of the two variables being correlated makes these results unreliable at best.

It is enlightning to highlight some of the preprocessing steps outlined in the previous section. To that end, the correlation between the nationwide number of strong and violent tornadoes and SST1 is examined. Figure 5a illustrates the periodic nature of SST1 (top) and how the application of the seasonal differencing filter removes it (bottom). Although it is not immediately obvious from the graph, the trend, too, has been removed.

Figures $5 \mathrm{~b}$ and $5 \mathrm{c}$ show the number of strong and violent tornadoes across the United States and the seasonally differenced values for each month, respectively. Given the wealth of information (or noise!) on these plots, it is not immediately obvious if there exists a correlation between the differenced SST1 (Fig. 5a, bottom) and the differenced number of strong and violent tornadoes (Fig. 5c). However, quantitatively one has $(\tau, z)=(-0.04,-1.42)$. Since $|z|=1.42$ falls below the critical value of 1.645 , conventionally one would conclude that the data do not provide sufficient evidence for rejecting the null hypothesis. As such, with $90 \%$ ( $\alpha$ $=0.1$ ) certainty the population $\tau$ may in fact be zero. However, according to the tabulated values of the normal distribution, $|z|=1.42$ implies that one can reject the null hypothesis with $84 \%(\alpha=0.1556)$ certainty. Therefore, there is some weak evidence for concluding that the number of strong and violent tornadoes is correlated with SST1.

The calculation of $\tau$ and $z$, and their interpretation, for the remaining correlations follows in a similar manner. Table 2 a shows the $(\tau, z)$ pairs for the correlation between SST and the number of tornadoes of all strength, and Table $2 \mathrm{~b}$ shows the correlation between SST and the number of tornadic days, both after the removal of the seasonal factor. Tables $3 \mathrm{a}$ and $3 \mathrm{~b}$ show the same set of correlations but for strong and violent 

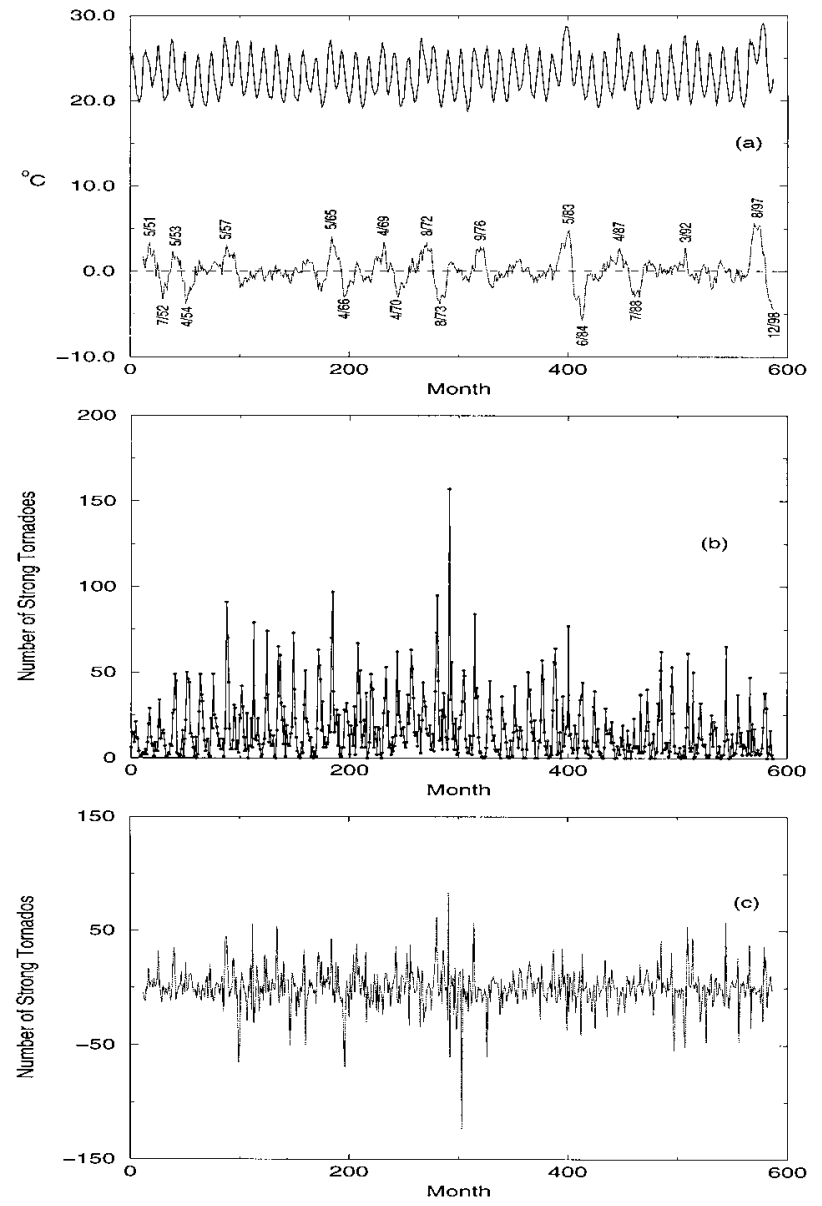

FIG. 5. (a) SST1 (top) and the seasonally differenced values thereof (bottom), and (b) the number of strong and violent tornadoes, and (c) its seasonally differenced values for every month.

tornadoes. As compared to the values in Table 1 (i.e., before filtering), it can be seen that the $z$ values have dropped dramatically. No longer is one faced with "large" correlations ( $\tau$ up to 0.21 ) of high significance ( $z$ up to 7.69). The pattern of the values in Tables 2 and 3 suggests a rich and complex interaction between the four zones in the Pacific and the various regions in the
United States. It is helpful to concentrate on $z$ values (i.e., significance), because they reflect the confidence in the $\tau$ values (i.e., correlation); in other words, a $\tau$, no matter how large, accompanied by a $|z|$ of, say, 0.001 is a dubious correlation, at best.

In Tables 2 and 3, it is worth pointing out that the correlations are generally too weak to have any forecast value since the $|\tau|$ values are all below 0.08 (i.e., with only $54 \%$ of the pairs being concordant). At the same time, the $z$ values vary over a wide range. Some of them are sufficiently large to suggest that there does exist a relationship between the corresponding SST and tornadic activity, albeit small.

Let us begin by examining the most significant (confident) correlations. For tornadoes of any strength (Table 2 ), when the measure of activity is the number of tornadoes (Table $2 \mathrm{a}$ ), the most significant of the correlations has $|z|=2.10$, insufficiently large to be considered significant at the $99 \%(\alpha=0.01)$ level, but sufficiently large to be considered statistically significant at the $96 \%$. On the other hand, when the measure of activity is the number of tornadic days (Table 2b), the most significant correlation $(|z|=2.91)$ is significant at the $99 \%$ level. In both cases, the most significant correlation is between SST2 and tornadic activity in region 2.

For strong and violent tornadoes (Table 3), and with the number of tornadoes as the measure of activity, the most significant result has $|z|=2.56$, almost large enough to be significant at the $99 \%$ level. When the measure of activity is the number of tornadic days, the most significant correlation has a $|z|$ of 2.68 , which is significant at the $99 \%$ level. Regardless of the measure of activity, the most significant correlation for strong and violent tornadoes is between SST4 and region 2.

All of these $z$ values are accompanied by the largest $\tau$ values in the respective tables. In other words, the strongest and most significant correlation for tornadoes of all strength is between region 2 (the northeast) and SST2 (the eastern equatorial Pacific). Strong and violent tornadoes are most strongly and significantly correlated between region 2 (the northeast) and SST4 (the central equatorial Pacific). However, it must be emphasized that

TABLE 2. Kendall's $\tau$ and its $z$ statistic, $(\tau, z)$, for the correlation between between SST and (a) the number of tornadoes, and (b) the number of tornadic days. The bold font indicates the most significant correlation in the respective table.

\begin{tabular}{|c|c|c|c|c|}
\hline & SST1 & SST2 & SST3 & SST4 \\
\hline \multicolumn{5}{|l|}{ (a) } \\
\hline Contiguous United States & $(-0.03,-1,10)$ & $(-0.05,-1.86)$ & $(-0.05,-1.86)$ & $(-0.04,-1.37)$ \\
\hline Region 1: Tornado Alley & $(-0.03,-1.21)$ & $(-0.04,-1.37)$ & $(-0.03,-1.18)$ & $(-0.01,-0.25)$ \\
\hline Region 2: Northeast United States & $(-0.04,-1.51)$ & $(-0.06,-2.10)$ & $(-0.05,-1.70)$ & $(-0.04,-1.41)$ \\
\hline Region 3: Southeast United States & $(-0.01,-0.48)$ & $(-0.03,-1.04)$ & $(-0.04,-1.41)$ & $(-0.06,-2.04)$ \\
\hline \multicolumn{5}{|l|}{ (b) } \\
\hline Contiguous United States & $(-0.02,-0.73)$ & $(-0.03,-0.91)$ & $(-0.02,-0.59)$ & $(-0.02,-0.60)$ \\
\hline Region 1: Tornado Alley & $(-0.02,-0.86)$ & $(-0.01,-0.33)$ & $(+0.01,+0.27)$ & $(+0.02,+0.87)$ \\
\hline Region 2: Northeast United States & $(-0.07,-2.62)$ & $(-0.08,-2.91)$ & $(-0.07,-2.35)$ & $(-0.05,-1.73)$ \\
\hline Region 3: Southeast United States & $(-0.01,-0.39)$ & $(-0.01,-0.20)$ & $(-0.004,-0.14)$ & $(-0.02,-0.65)$ \\
\hline
\end{tabular}


TABLE 3. Kendall's $\tau$ and its $z$ statistic, $(\tau, z)$, for the correlation between SST and (a) the number of strong and violent tornadoes, and (b) the number of days with strong and violent tornadoes. The bold font indicates the most significant correlation in the respective table.

\begin{tabular}{lcccc}
\hline \hline & SST1 & SST2 & SST3 & SST4 \\
\hline (a) & & & & \\
$\quad$ Contiguous United States & $(-0.04,-1.42)$ & $(-0.06,-2.12)$ & $(-0.06,-2.28)$ & $(-0.07,-2.34)$ \\
$\quad$ Region 1: Tornado Alley & $(-0.07,-2.42)$ & $(-0.06,-2.30)$ & $(-0.05,-1.94)$ & $(-0.03,-1.22)$ \\
$\quad$ Region 2: Northeast United States & $(-0.04,-1.50)$ & $(-0.06,-2.30)$ & $(-0.06,-2.21)$ & $(-\mathbf{0 . 0 7},-\mathbf{2 . 5 6 )}$ \\
$\quad$ Region 3: Southeast United States & $(+0.01,+0.47)$ & $(-0.001,-0.02)$ & $(-0.02,-0.75)$ & $(-0.04,-1.45)$ \\
(b) & & & & $(-0.06,-2.15)$ \\
Contiguous United States & $(-0.05,-1.63)$ & $(-0.05,-1.68)$ & $(-0.05,-1.80)$ & $(-0.06,-2.03)$ \\
$\quad$ Region 1: Tornado Alley & $(-0.02,-0.57)$ & $(-0.03,-1.11)$ & $(-0.04,-1.48)$ & $(-\mathbf{0 . 0 7},-\mathbf{2 . 6 8})$ \\
$\quad$ Region 2: Northeast United States & $(-0.04,-1.33)$ & $(-0.06,-2.01)$ & $(-0.06,-2.05)$ & $(-0.01,-0.50)$ \\
$\quad$ Region 3: Southeast United States & $(+0.01,+0.27)$ & $(+0.01,+0.39)$ & $(-0.001,-0.03)$ &
\end{tabular}

the strongest of these $\tau$ 's still has a magnitude of only 0.08 .

In general, Table 2 suggests that the correlation between any SST and tornadic activity in the United States is mostly not significant. As such, all the corresponding $\tau$ values are statistically indistinguishable from zero. Of the regions considered, region 2 (northeastern United States) generally has the most significant correlations with any of the SSTs. Regions 1 and 3 are generally uncorrelated with any of the SSTs, with the exception of SST4 whose correlation with the number of tornadoes in region 3 is modestly significant $(\tau, z)=$ $(-0.06,-2.04)$.

None of the four SSTs in the Pacific consistently have the most significant correlations with general tornado activity in the United States. For instance, SST4 has the most significance when correlated with all tornadoes in region 3 , but with tornadic days in region 2 .

As for strong and violent tornadoes (Table 3), a general pattern that emerges is higher significance levels when activity is gauged in terms of the number of tornadoes (in contrast to the case of tornadoes of any strength). Another pattern is that region 3 is generally uncorrelated with any of the SSTs. Region 1 shows some correlation with SST1 and SST2 when activity is measured with the number of tornadoes, and no correlation when activity is gauged with the number of tornadic days. The latter in region 1 is somewhat correlated with SST4. SST4 typically has the most significant correlations when either strong and violent tornadoes or strong and violent tornado days are considered.

Note that with few exceptions, all of the $\tau$ values are negative. A positive value would be suggestive of a positive correlation with El Niño (or a negative correlation with La Niña), while a negative value would suggest a negative correlation with El Niño (or a positive correlation with La Niña). The exceptions have $z$ values too small to be considered statistically significant. According to the data, El Niño seems to have no positive correlation with the tornadic activity occurring anywhere in the United States. In other words, when a (somewhat) significant correlation does exist, it is quite weak and mostly between tornadic activity and La Niña.

\section{Summary and discussion}

An examination of $49 \mathrm{yr}$ of data (i.e., 588 months) reveals that there appears to exist a statistically significant, but very small, correlation between sea surface temperature anomalies in the Pacific Ocean and tornadic activity in the United States. The strength and significance of the correlations depends on the zones over which the sea surface temperatures are assessed and the geographic region of the United States. In general, the correlations are negative, suggesting that a higher frequency of tornadoes and tornadic days is associated with cooler sea surface temperatures (La Niña). For instance, the sea surface temperature in the eastern equatorial Pacific (SST2) and the number of tornadic days in the northeastern United States (region 2) exhibit a negative correlation significant at the $\alpha=0.0018$ level, corresponding (loosely) to a $99.82 \%$ probability that the null hypothesis (of zero correlation) is false. Also, the sea surface temperature in the central equatorial Pacific (SST4) is correlated with strong and violent tornadic activity in the same geographic region of the United States at the $+99.5 \%$ level.

Regardless of the strength and the statistical significance of the correlations they do reveal a pattern that is partially explainable. For instance, while the atmospheric processes that control the formation of thunderstorms capable of producing tornadoes are dominated by local- to subregional-scale conditions, the general circulation does play a modulation role. For instance, the cooling of the central equatorial Pacific leads to the development of a global circulation pattern in which the midlatitude jet stream crosses the northeastern United States (Hoerling et al. 1997), which in turn will enhance that area's potential for the development of supercell thunderstorms and strong and violent tornadoes. The small degree of correlation $(\tau=-0.08)$ indicates that many other factors play a role in determining if tornadoes do indeed occur, but the correlation is highly significant. According to this model, over the long term more strong and violent tornadoes will be observed in the northeastern United States during La Niña months than during El Niño months; but for any specific La 

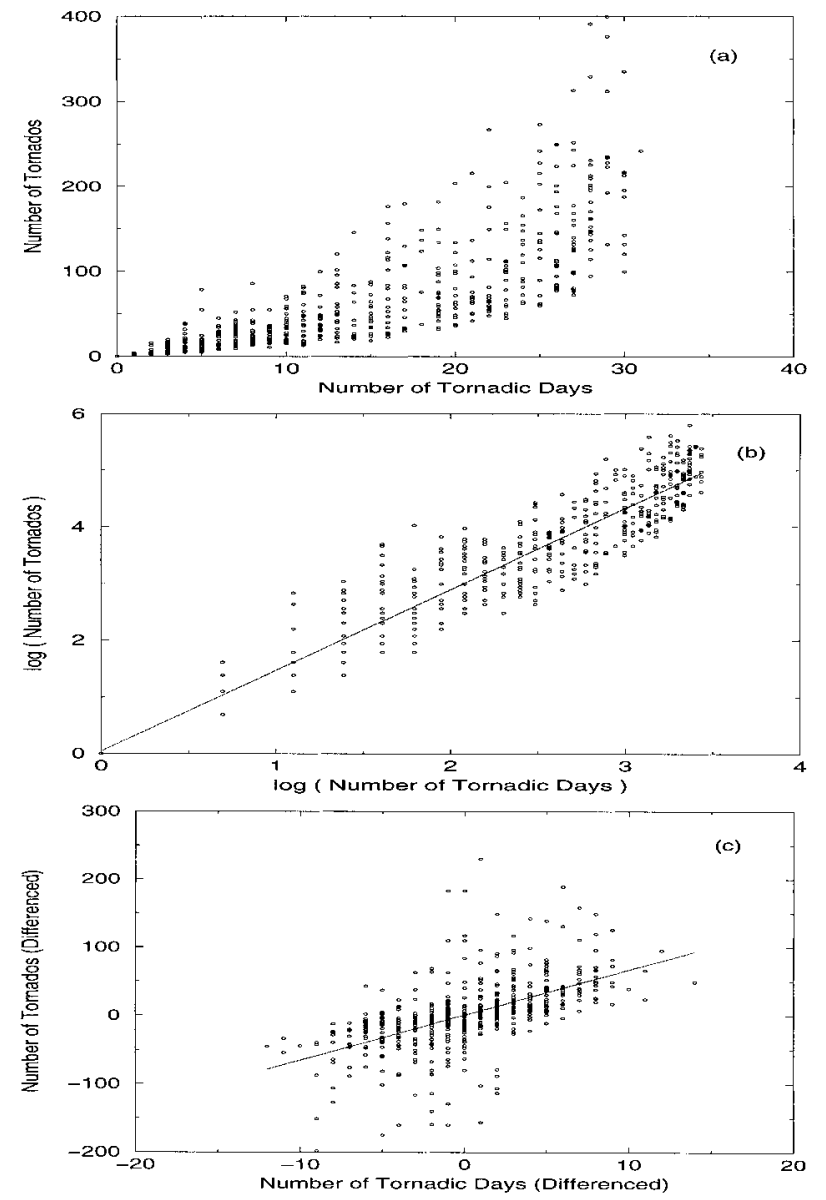

FIG. 6. The scatterplot of (a) the number of tornadoes per month vs the number of tornadic days per month, (b) on a log-log plot, and (c) on a linear plot, but seasonally differenced. The straight lines are the regression fits to the data.

Niña month there is only a $53 \%$ chance that this will occur.

One may ask if the two measures of tornadic activity (i.e., the number of tornadoes per month, and the number of tornadic days per month) are similar to one another. In particular, are the two measures correlated? The scatterplot of the two quantities is shown in Fig. 6a. Evidently, there exists some nonlinear relation between them. In fact, as shown in Fig. 6b, that relationship on a log-log plot is nearly linear, $y=0.59 x+0.35$, with a linear correlation coefficient of $r=0.92$. In other words, there is a strong power-law relation $(y=$ $\left.1.42 x^{0.59}\right)$ between the number of tornadoes per month and the number of tornadic days per month. Although the existence of a power law is interesting, it appears to be primarily due to the periodic nature of the two quantities; specifically, if the autocorrelation in the two is filtered out by seasonal differencing, the filtered quantities appear to be mostly uncorrelated (Fig. 6c). This implies that the two measures gauge different facets of tornadic activity. As such, the results of the analysis of the correlation between SST and tornadic activity are expected to be independent of the two specific measures of the latter.

Similarly, one may inquire into the correlation between the four SSTs. Needless to say, the correlations are meaningful only after the seasonal cycle has been removed. The (symmetric) correlation matrix for the four seasonally differenced SSTs is

$$
\left(\begin{array}{llll}
1.00 & 0.85 & 0.72 & 0.57 \\
& 1.00 & 0.96 & 0.78 \\
& & 1.00 & 0.89 \\
& & & 1.00
\end{array}\right) .
$$

Clearly, not all four of the SSTs are mutually independent. The strengths vary from $r=0.96$ between SST2 and SST3, to $r=0.57$ between SST1 and SST4. The relative strengths of the various correlations appear to reflect the relative position and the amount of overlap between the various regions in the Pacific.

A question that arises is that of the robustness of the measure $\tau$ as far as outliers are concerned. In other words, how sensitively does $\tau$ (and its corresponding $z$ value) depend on, say, the high peak (3 April 1974) appearing in Fig. 2b. The best way to address that question is the brute force way; in particular, the highest peak in that figure corresponds to 157 strong and violent tornadoes. That number was artificially replaced by the sample average number of strong and violent tornadoes (i.e., 66.6), and $\tau$ and $z$ were recomputed. For example, the nationwide values of $\tau$ and $z$ for the four regions were found to be $(-0.04,-1.49),(-0.06,-2.11)$, $(-0.06,-2.22)$, and $(-0.06,-2.21)$, respectively. Compared to the actual results (i.e., first line of Table 3 ), the $\tau$ and the $z$ values are mostly unaffected. This means the correlations as gauged by $\tau$ and $z$ are relatively robust with respect to outliers (or, at least, with respect to a single outlier).

How sensitively do the findings in this paper depend on sample size? In other words, are 49 yr of data sufficient to justify the conclusions? There are many ways to answer this question, and producing $z$ values (as we have done above) is one of them, because they indicate how generalizable are the results to the general population. Another way to address the question is to examine the time dependence of the correlations. For instance, what are $\tau$ and $z$ for only the first $5 \mathrm{yr}$ of data? The first $10 \mathrm{yr}$, etc.? The result is shown in Fig. 7, wherein $\tau$ and $z$ between the number of strong and violent tornadoes in region 2 and SST4 are computed for the first $5,10,15, \ldots, 45$, and 49 yr (i.e., the entire dataset). The figure shows that for the first $15 \mathrm{yr}$, the correlation is actually positive, but not significantly so (at the 95\% level, shown by the dashed, horizontal lines). Even though the correlation turns negative after about $15 \mathrm{yr}$ of data, it continues to be nonsignificant up until about 35-40 yr of data have been accumulated. For the larger data samples (i.e., more years), the plot 


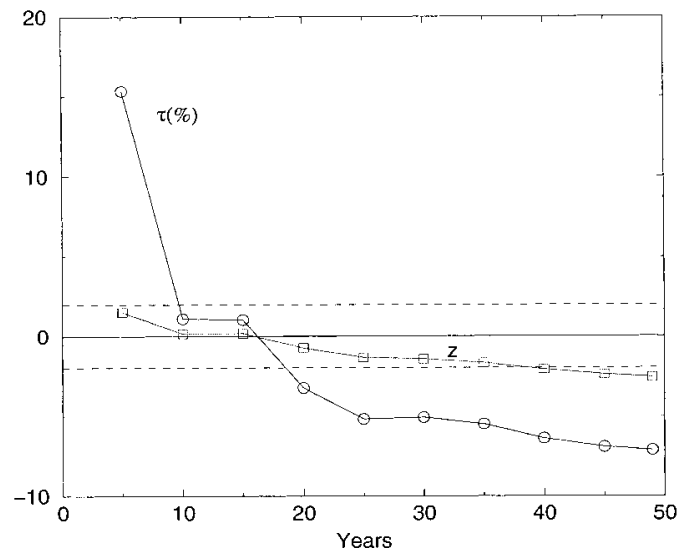

FIG. 7. $100 \times \tau$ (circles) and $z$ (squares) between SST4 and strong and violent tornadoes in region 2, as a function of sample size (years). The region in between the dashed lines is the $95 \%$ confidence interval.

does not show any signs of a reversal in the sign of $\tau$ (or $z$ ). Therefore, it appears that the correlation will continue to be negative and significant (at least into the near future). It is also important to note the asymptotic nature of the $\tau$ curve with increasing time. In other words, $\tau$ levels off for longer time spans to a value of about -0.08 , suggesting that it is unlikely that the correlation will diminish as the data grow in size. At 35$40 \mathrm{yr}, z$ crosses out of the $95 \%$ confidence band and continues to grow in the negative direction. In other words, since about 1980 there have existed sufficient data to reject the null hypothesis (that there is no correlation between SST and tornadic activity) with $95 \%$ confidence. In fact, with $49 \mathrm{yr}$ of data, that confidence is increased to nearly $99 \%$.

It is peculiar that the significance of the correlation based on only $5 \mathrm{yr}$ of data is comparable to that with nearly 30 years of data. One could argue that the trend displayed in Fig. 7 is "real," in that the correlation may in fact be a function of time. This would imply that the climatology itself has been changing over time. An alternative interpretation is to dismiss the positive correlation in the early years based on the fact that the $z$ values reflect less than $95 \%$ confidence. However, it is also possible that this behavior is an artifact of the seasonal differencing that was performed to filter the data. In short, more data and/or more analysis may be required to fully address this phenomenon.

Let us mention, in passing, that the plot of the seasonally differenced SST1 (Fig. 5a) allows for an identification of the unusually warm or cold months, that is, the SST1 anomalies, or the El Niño and La Niña months. The dates (month/year) of both the warm and the cold months have been printed on the graph. These dates can be taken as a definition of El Niño and La Niña months, respectively. However, as mentioned in the introduction, such a definition is not unique because other SSTs yield somewhat different warm/cold months.

One may note that treating each of the four SSTs individually constitutes four univariate models. In principle, it is possible to develop a multiple regression model that simultaneously relates tornadic activity to all four SSTs. Although such a model is apt to outperform any of the univariate models in predicting tornadic activity, it will not be able to explain the relationship between tornadic activity and the SSTs because of the correlations between the four SSTs; any collinearity in the independent variables in a multiple regression model renders the regression coefficients (i.e., slopes) entirely meaningless (Draper and Smith 1981; Tacq 1997; Marzban et al. 1999).

In the present analysis, the correlation between SST (anomalies) and the number of tornadoes is tacitly assumed to be an "instantaneous" one. This assumption is not strictly valid. The response of the general circulation to development/dissipation of a ridge/trough takes place as Rossby wave motion (Hoskins and Karoly 1981). Circulation changes over eastern North America in response to forcing over the west-central equatorial Pacific should occur on a timescale of several days. It is this time factor that argued for the use of monthly rather than annual statistics. One could further test the monthly correlation at some lagged interval. In other words, it is possible that a change in one SST may cause a change in tornadic activity at some later time. This possibility is currently under consideration.

Finally, it must be emphasized that the conclusions reported herein are contingent on two important quantities: the quality of the data and of the filter. The former can be improved upon by enlarging the sample size. This can be done in at least two ways; accumulating data over a longer length of time, or by considering a shorter unit of time, for example, days (rather than months). Although an analysis based on a daily unit of time will increase the sample size it will most likely also increase the noise in the data and so may not be fruitful. The second contingency is more readily tractable, in that there exist a plethora of filters with different properties that may be applied. In particular, the filter employed herein tends to filter out cyclic and strictly linear trends. It is possible that a filter different from the one considered here may expose a different pattern of correlations. Other filters are currently being tested.

Acknowledgments. Harold Brooks, V. Lakshmanan, and Arthur Witt of the National Severe Storms Laboratory (NSSL) and Russell Schneider of the Storm Prediction Center (SPC) are acknowledged for numerous useful discussions and suggestions. Discussions with Dave Schultz (NSSL) and Ants Leetmaa (Climate Prediction Center) on the response time of the circulation to SST changes were most instructive. Tom Smith (CPC) provided us with the SST values, and Joan O'Bannon (NSSL/CIMMS) prepared Fig. 1. We also thank William Kessler and Dennis Moore (PMEL) for pointing out an error in an earlier version of this paper. Finally, we are grateful to the anonymous reviewers for providing in- 
sight into the evolution of the definition of the terms El Niño and La Niña.

\section{APPENDIX \\ Kendall's $\tau$}

In this appendix a simple example is considered wherein Kendall's $\tau$ can be computed manually. Press et al. (1986) and Wilcox (1996) are both excellent references for learning more about Kendall's $\tau$ and other nonparametric measures of association. The basic advantage of nonparametric measures of association over parametric measures is that the former do not rely on any assumptions regarding the data. Why, then, are parametric measures used at all? Because they are associated with statistical models that can be used for objective prediction. For example, Pearson's linear correlation coefficient, $r$, can be used in a linear regression equation. Nonparametric measures are not associated with any model and so are used only for testing the hypothesis of whether or not a relationship exists at all.

Consider the following data on the pair of variables $(x, y):(1942,61.0), \quad(1943,60.6), \quad(1944,59.8)$, $(1945,60.3)$. These are actually the annual mean temperatures in Raleigh, North Carolina, for the years 1942-45. Note that 1942 was warmer than 1943, 1944, and 1945. Similarly, 1943 was warmer than 1944 and 1945, while 1944 was colder than 1945 . So, when considered in pairs, in 5 out of the 6 possible pairs, an earlier year is warmer than a later year. That means five discordant pairs and one concordant pair. Then, Eq. (1) implies that $\tau=2(1-5) /[4(4-1)]=-2 / 3=-0.67$. This value of $\tau$ suggests a cooling trend. But is it significantly different from 0? Equation (2) gives $z=$ $-(2 / 3) \sqrt{(54 / 13)}=-1.36$, according to which, based on tabulated values of $z$, there is only a $91 \%$ probability that the population value of $\tau$ is nonzero. Given such relatively low probability, one may not be inclined to reject the null hypothesis of $\tau=0$. In short, the data do not provide sufficient evidence to support the hypothesis that Raleigh, North Carolina, was cooling over the years 1942-45. Traditionally, a probability of $95 \%$ or even $99 \%$ would be demanded in order to reject the null hypothesis $\tau=0$.

\section{REFERENCES}

Agee, E., and S. Zurn-Birkhimer, 1998: Variations in USA tornado occurrences during El Niño and La Niña. Preprints, 19th Conf. on Severe Local Storms, Minneapolis, MN, Amer. Meteor. Soc., 287-290.

Bove, M. C., 1998: Impacts of ENSO on United States tornado ac- tivity. Preprints, Ninth Symp. on Global Change Studies, Phoenix, AZ, Amer. Meteor. Soc., 199-202.

Browning, P., 1998: ENSO related severe thunderstorm climatology of northwest Missouri. Preprints, 19th Conf. on Severe Local Storms, Minneapolis, MN, Amer. Meteor. Soc., 291-292.

Climate Prediction Center, Vol. 99, 1999: Climate Diagnostics Bulletin. Vol. 99, 2, No. 2, 81 pp.

Draper, N. R., and H. Smith, 1981: Applied Regression Analysis. John Wiley and Sons, 709 pp.

Grazulis, T. P., J. T. Schaefer, and R. R. Abbey, 1993: Advances in tornado climatology, hazards, and risk assessment since Tornado Symposium II. The Tornado: Its Structure, Dynamics, Prediction, and Hazards, Geophys. Monogr., No. 79, Amer. Geophys. Union, 409-426.

Hagemeyer, B. C., 1998: Significant extratropical tornado occurrences in Florida during strong El Niño and strong La Niña events. Preprints, 19th Conf. on Severe Local Storms, Minneapolis, MN, Amer. Meteor. Soc., 412-415.

Halpert, M. S., and C. F. Ropelewski, 1987: Global and regional scale precipitation patterns associated with the El Niño/Southern Oscillation. Mon. Wea. Rev., 115, 1606-1626.

- and — 1992: Surface temperature patterns associated with the Southern Oscillation. J. Climate, 5, 577-593.

Hamming, R. W., 1983: Digital Filters. Prentice-Hall, Inc., 257 pp.

Hoerling, M. P., A. Kumar, and M. Zhong, 1997: El Niño, La Niña, and the nonlinearity of their teleconnections. J. Climate, 10, $1769-1786$.

Hoskins, B. J., and D. J. Karoly, 1981: The steady linear response of a spherical atmosphere to thermal and orographic forcing. $J$. Atmos. Sci., 38, 1179-1196.

Jenkins, G. M., and D. G. Watts, 1969: Spectral Analysis and Its Applications. Holden-Day, 525 pp.

Marzban, C., E. D. Mitchell, and G. Stumpf, 1999: The notion of "best predictors:" An application to tornado prediction. Wea. Forecasting, 14, 1007-1016.

Masters, T., 1995: Neural, Novel \& Hybrid Algorithms for Time Series Prediction. John Wiley and Sons, $514 \mathrm{pp}$.

Press, W. H., B. P. Flannery, S. A., Teukolsky, and W. T. Vetterling, 1986: Numerical Recipes: The Art of Scientific Computing. Cambridge University Press, $818 \mathrm{pp}$.

Ramage, C. S., 1975: Preliminary discussion of the meteorology of the 1972-73 El Niño. Bull. Amer. Meteor. Soc., 56, 234-242.

Rasmusson, E. M., and T. H. Carpenter, 1982: Variations in tropical sea surface temperature and surface wind fields associated with the Southern Oscillation/El Niño. Mon. Wea. Rev., 110, 354384.

Reynolds, R. W., and T. M. Smith, 1994: Improved global sea surface temperature analysis using optimum interpolation. J. Climate, 7, 929-948.

Ropelewski, C. F, and M. S. Halpert, 1989: Precipitation patterns associated with the high index phase of the Southern Oscillation. J. Climate, 2, 577-284.

Schaefer, J. T., and F. B. Tatom, 1998: The relationship between El Niño, La Niña, and United States tornadoes. Preprints, 19th Conf. on Severe Local Storms, Minneapolis, MN, Amer. Meteor. Soc., 416-419.

, and R. Edwards, 1999: The SPC Tornado/Severe Thunderstorm Database. Preprints, 11th Conf. on Applied Climatology, Dallas, TX, Amer. Meteor. Soc., 215-220.

Tacq, J., 1997: Multivariate Analysis Techniques in Social Science Research. Sage Publications, $411 \mathrm{pp}$.

Wilcox, R. R., 1996: Statistics for the Social Sciences. Academic Press, $454 \mathrm{pp}$. 